\section{ENGINEERED NATURAL KILLER CELLS REACTIVELY BLOCK TIGIT AND CD73 IN THE GBM MICROENVIRONMENT}

Kyle Lupo*, Sandro Matosevic. Purdue University, Lafayette, IN, USA

Background Natural killer (NK) cells have emerged as promising effectors to target GBM and other solid tumors through genetic modifications and ex vivo manipulation. However, immunosuppressive conditions within the tumor microenvironment (TME) and interactions between NK cell activating and inhibitory receptors further complicate NK cell-based treatments. In particular, the $\mathrm{T}$ cell immunoreceptor with $\mathrm{Ig}$ and ITIM domains (TIGIT) is expressed on NK cells and interacts with CD155 to induce immunosuppression of NK cell cytolytic functions. ${ }^{1}{ }^{2}$ Although CD155 also binds with activating receptors DNAM-1 and CD96 on NK cells, spurring NK cell activity, TIGIT has predominantly been reported as having an inhibitory effect on NK cells. ${ }^{3-5}$ Further, tumor cells release of high levels of ATP extracellularly. While intracellular ATP is necessary for cell metabolism, extracellular ATP is converted into adenosine (ADO) by ectonucleotidases CD39 and CD73, both overexpressed on GBM and other solid tumors. ${ }^{6}$ Extracellular ADO induces immunometabolic suppression of NK cells through binding with $\mathrm{A} 2 \mathrm{~A}$ adenosine receptors (A2ARs) on NK cells, suppressing cytokine secretion, proliferation, and other functional activities. ${ }^{7-9}$ We found that TIGIT and CD73 are effective combination targets in GBM for both primary and iPSC-derived NK cells.

Methods In order to effectively target immunometabolic reprogramming induced by CD73-produced adenosine and the immunosuppressive TIGIT-CD155 axis, we have engineered NK cells to concomitantly target CD155 and CD73-induced immunosuppression on GBM using a tumor-responsive genetic construct based on the synNotch signaling system. The construct is capable of blocking the immunosuppressive CD155/ TIGIT interaction, and, upon binding, release a CD73-blocking scFv to inhibit the accumulation of extracellular ADO and mitigate immunosuppression of NK cells. Such localized response enhances specificity and reduces off-target effects of NK-based targeting.

Results Primary NK cells and iPSC-derived NK cells were successfully engineered to express the synthetic TIGIT-synNotch construct, measured through expression of TIGIT. To evaluate the functionality of engineered NK cells against GBM targets, we tested the cytotoxicity of our engineered NK cells against a primary, patient-derived GBM cell line, GBM43. Overall, cytolytic function of engineered NK cells against GBM was significantly higher than that of non-engineered NK cells, with or without CD73 (10 ug/mL) and TIGIT $(50 \mathrm{ug} / \mathrm{mL})$ antibodies, for E:T ratios of 5:1 and 10:1, demonstrating the functional efficacy of our genetic construct.

Conclusions Overall, we have shown that co-targeting CD155 and $\mathrm{CD} 73$ in a localized, responsive manner can dampen immunosuppression and significantly enhance the killing potential of engineered NK cells against aggressive patient-derived GBM tumors.

\section{REFERENCES}

1. Zhang B, et al. Immunoreceptor TIGIT inhibits the cytotoxicity of human cytokineinduced killer cells by interacting with CD155. Cancer Immunol Immunother 2016:65:305-314.

2. Lupo KB \& Matosevic S. CD155 immunoregulation as a target for natural killer cell immunotherapy in glioblastoma. J Hematol Oncol 2020;13:76.
3. Hung AL, et al. TIGIT and PD-1 dual checkpoint blockade enhances antitumor immunity and survival in GBM. Oncolmmunology 2018; e1466769. doi:10.1080/ 2162402X.2018.1466769.

4. Mahnke K \& Enk, AH. TIGIT-CD155 Interactions in Melanoma: A Novel Co-Inhibitory Pathway with Potential for Clinical Intervention. Journal of Investigative Dermatology 2016; 136, 9-11.

5. Stanietsky N, et al. Mouse TIGIT inhibits NK-cell cytotoxicity upon interaction with PVR: Innate immunity. Eur J Immunol 2013; 43:2138-2150.

6. Chambers $\mathrm{AM}$, et al. Adenosinergic Signaling Alters Natural Killer Cell Functional Responses. Front Immunol 2018;9:2533.

7. Chambers AM, Lupo KB \& Matosevic S. Tumor Microenvironment-Induced Immunometabolic Reprogramming of Natural Killer Cells. Front Immunol 2018;9:2517.

8. Chambers AM. et al. Adenosinergic Signaling Alters Natural Killer Cell Functional Responses. Front Immunol 2018;9:2533.

9. Wang J, Lupo KB, Chambers AM \& Matosevic S. Purinergic targeting enhances immunotherapy of CD73+ solid tumors with piggyBac-engineered chimeric antigen receptor natural killer cells. J Immunotherapy Cancer 2018;6:136.

Ethics Approval Primary human NK cells were obtained from healthy adult donors approved under Purdue University's Institutional Review Board (IRB) (IRB-approved protocol \#1804020540). Donors gave written informed consent prior to taking part in the study.

http://dx.doi.org/10.1136/jitc-2021-SITC2021.130 